\title{
Comparing determinants of physical activity in Puerto Rican, Mexican-American, and non-Hispanic white breast cancer survivors
}

\author{
Daniel C Hughes ${ }^{1 *}$, Maribel Tirado-Gomez² , Liliana Vallejo ${ }^{3}$, Velda Gonzalez², Rose A Treviño-Whitaker', \\ Gabriela Villanueva ${ }^{1}$ and Karen Basen-Engquist ${ }^{3}$
}

\begin{abstract}
Purpose: Physical activity (PA) has a myriad of benefits for breast cancer survivors, including a reduced risk of cancer recurrence. Latinas are less physically active than are women in the general population and little is known about Latina breast cancer survivors' levels of PA or their beliefs related to PA. We conducted a survey of 50 Puerto Rican (PR), 50 Mexican-American (MA) and 50 non-Hispanic white (NHW) breast cancer survivors to investigate similarities and differences in PA and social cognitive theory (SCT) constructs associated with PA.

Methods: We collected information on current PA using the Godin Leisure Time Exercise Questionnaire (GLTEQ); comorbidities; anthropometric measures of body mass index [BMI $\left.\left(\mathrm{kg} / \mathrm{m}^{2}\right)\right]$ and waist-to-hip (W:H) ratio; and SCT measures, including exercise self-efficacy, exercise barriers self-efficacy, modeling and social support from friends and family. Descriptive statistics, one-way analysis of variance of differences between groups and regression models of the predictors of PA were performed.

Results: Survivors from the three groups were similar in age $(M=56.8, S D=11.0), B M I(M=29.0, S D=5.7)$ and co-morbidity ( $M=2.09$, SD $=1.69)$. Survivors differed in PA $(p<0.001)$, self-efficacy $(p=0.05)$, modeling $(p=0.03)$ and social support from family $(p=0.05)$. Social support from family member and exercise barriers self-efficacy were predictors of PA.

Conclusions: Consistent with published studies, Hispanic breast cancer survivors self-report that they are less physically active than are non-Hispanic whites. SCT variables associated with PA differ among Hispanic subgroups and nonHispanic whites. Further research is warranted in order to understand determinants of physical activity for specific ethnic breast cancer survivors.
\end{abstract}

Keywords: Hispanics, Social cognitive theory, Exercise, Breast cancer

\section{Background}

The US now has more than 12 million cancer survivors (American Cancer Society 2012) and at least 1.8 million of those are Hispanic (American Cancer Society 2009). Hispanics are the most rapidly growing ethnicity in the United States (United States Census Bureau 2000), and breast cancer is the leading cause of cancer death among Hispanic

\footnotetext{
*Correspondence: hughesdc@uthscsa.edu

${ }^{1}$ Institute for Health Promotion Research, The University of Texas Health Science Center at San Antonio, 7411 John Smith Drive, Suite 1000, San Antonio, TX 78229, USA

Full list of author information is available at the end of the article
}

women (American Cancer Society 2009). In Puerto Rico it accounts for approximately 34\% of all female cancers and is the most common female malignancy diagnosed (Puerto Rico Central Cancer Registry 2007).

Research continues to document the benefits of exercise for breast cancer survivors, including improved fitness, physical functioning, fatigue and emotional wellbeing (Courneya 2003; Courneya et al. 2003; Segal et al. 2001; Pinto et al. 2005). Moreover, cohort studies have shown a decreased risk of breast cancer recurrence and reduced mortality among survivors who are more active (Holmes et al. 2005; Irwin et al. 2011; Ballard-Barbash

\section{Springer}


et al. 2012; Patterson et al. 2010). However, only a small percentage of survivors are active at levels consistent with public health guidelines (Schmitz et al. 2010). Like others who experience cancer, many breast cancer survivors who were not active before diagnosis will remain inactive, and those who were active often do not return to their previous level of activity (Schmitz et al. 2010). In fact, approximately four of every five breast cancer survivors do not meet national exercise recommendations at 10 years post-diagnosis (Mason et al. 2013). Little is known about the physical activity levels of Hispanic breast cancer survivors, although some studies indicate that in the general US population, Latinas report higher rates of inactivity than do non-Hispanic white women (Castro et al. 1999; Crespo et al. 2000).

Although the benefits of physical activity for cancer survivors continue to be documented and are becoming better understood, research is needed to identify interventions that encourage cancer survivors to begin and/or maintain consistent exercise patterns. Intervention strategies for healthy and predominately non-Hispanic white populations may not apply to members of specific ethnocultural populations that have experienced cancer and who may face additional cultural factors that affect physical activity. Furthermore, diversity within Hispanic populations may also affect the likelihood that an individual will pursue physical activity after cancer diagnosis and treatment. Differences among Hispanic groups, including race, country of origin, levels of acculturation, English language proficiency, and other cultural variables, may affect health-promoting behaviors, including exercise.

Therefore we believe that investigating the key variables that affect physical activity adoption and maintenance within specific cultural contexts of Hispanic breast cancer survivor groups is a high priority for behavioral research. Understanding the knowledge, attitudes and barriers that surround physical activity among Hispanic groups is fundamental in the development of culturally appropriate exercise interventions. To provide formative data for adapting a culturally sensitive social cognitive theory (SCT)-based exercise intervention, we conducted a cross-sectional survey to investigate similarities and differences in SCT, co-morbidity and anthropometric variables associated with current level of physical activity in Puerto Rican, Mexican-American and non-Hispanic white breast cancer survivors.

\section{Methods}

A sample of 150 breast cancer survivors completed an interviewer-guided survey. Fifty Puerto-Rican (PR) participants completed the survey in San Juan Puerto Rico at the Oncologic Hospital Dr. Isaac Gonzalez Martinez. Fifty Mexican-American (MA) and 50 non-Hispanic white (NHW) breast cancer survivors completed the survey at The University of Texas M.D. Anderson Cancer Center (UTMDACC). The research protocol was approved by the institutional review board of UTMDACC. In Puerto Rico, the research protocol was approved by the University of Puerto Rico Medical Sciences and the institutional review board of the Oncologic Hospital Dr. Isaac Gonzalez Martinez. Participants were invited to participate in the study if they: (1) had a diagnosis of invasive breast cancer or ductal carcinoma in situ; (2) were older than 21 years (Puerto Rico) or older than 18 years (UTMDACC) of age; (3) had finished their chemotherapy and/or radiotherapy at least four months before the date of the survey (hormonal therapy was allowed); and, (4) had no evidence of metastatic disease.

For Puerto Rican participants, the clinical study coordinator reviewed the medical charts of patients attending the outpatient clinic in the Oncologic Hospital Dr. Isaac Gonzalez Martinez clinic and then met with potential participants who met the inclusion criteria at the clinic. Participants who expressed interest in participating received an informative sheet describing the study's purpose and procedures. Verbal consent was obtained before data were collected. Participants filled out the questionnaires in the presence of the clinical study coordinator who was available to answer any questions. Surveys were conducted in Spanish.

At UTMDACC, women were recruited through the Breast Oncology Clinic during scheduled appointments or from Houston community breast cancer support groups. From UTMDACC patient data records, a list of women who met the inclusion criteria and were returning for follow-up care was generated. These women received a letter in the mail that provided information about the study and with a number to call if not interested. If no call was received, a bilingual research coordinator called and/or met the patient at her follow-up appointment to explain the study, obtain verbal informed consent and schedule an interview. Participants at UTMDACC were asked whether they preferred Spanish or English. The survey questions were interviewer guided. To help facilitate the interviewer-guided response, participants were given response cards specific to each section of the questionnaire, (e.g., Likert scale choices in large print). The research coordinator explained the use of response cards. Participants were asked to give their initial response after each item was read aloud. For both UPRCC and MDACC, the survey included identical questions on demographic/personal information, health history, level of physical activity and SCT variables.

Demographic and personal information included living location; self-reported ethnicity/race; education; marital status; self-reported height and weight (used for BMI 
calculation) and medical history. A co-morbidity index was calculated using responses from the medical history information. A total of 17 items were included: diagnosis of a heart attack, heart failure, heart condition, circulation problems, blood clots, hypertension, stroke, lung problems, diabetes, kidney problems, rheumatoid arthritis, osteoarthritis, anemia, thyroid problems, neuropathy, fibromyalgia and hepatitis. The co-morbidity index was calculated by adding the number of individual responses marked as "Yes" (with a possible range of scores of 0-17). Participants also were whether they experienced any secondary cancers and whether they had been given a diagnosis of lymphedema (The information on secondary cancers and lymphedema was noted but was treated separately from the co-morbidity index).

Current level of physical activity (PA) was assessed with The Godin Leisure Time Exercise Questionnaire (GLTEQ) (Godin and Shephard 1985). The GLTEQ measures leisure time exercise behaviors for a typical week. Participants completed a four-item questionnaire of usual leisure-time exercise habits. As an example, a typical question regarding strenuous activity was: "Considering a 7-day period (a week), how many times, on the average, do you do the following kinds of exercise for more than 15 min during your free time: a) strenuous exercise (hearts beats rapidly [i.e., running, jogging, football, soccer, squash, basketball, cross country skiing, judo, roller skating, vigorous long distance bicycling.])". The weekly frequency of each participant's strenuous, moderate and light activities were multiplied by 9,5 and 3 , respectively which is an approximate metabolic equivalents (MET) value for that level of activity. MET is the metabolic equivalent of the level of energy consumption for a body at rest; the higher a MET value, the higher the energy requirement. The MET value was used with the individual's self-reported frequency to calculate a weekly activity score [weekly activity score $=(9 \times$ "strenuous") $+(5 \times$ "moderate" $)+(3 \times$ "light")]. The GLTEQ has been validated as a means of discriminating among levels of PA and has compared well with other measures of exercise, fitness indices, and physical activity monitors (Godin and Shephard 1985).

SCT variables of self-efficacy, modeling and social support were included in the survey. Self-efficacy was measured with a questionnaire based on one used by McAuley (1993); (McAuley et al. 1994, 2003; Duncan and McAuley 1993) that assesses confidence in sustaining various durations of a specific exercise, in our case, walking. A typical question was: "How confident are you that you can... Walk briskly without stopping for $5 \mathrm{~min}$ ?" The responses range from $1=$ "not at all confident" to $5=$ "extremely confident". The range of the time frame we used for minutes of walking was from 2 min to $1 \mathrm{~h}(2,5,10,20,30$,
$45 \mathrm{~min}, 1 \mathrm{~h}$ ). Responses to the seven individual items were summed to obtain an overall score with a possible range of 7-35. Internal consistency (Cronbach's alpha) for our survey was $r=0.96$.

To measure exercise barriers self-efficacy we adapted a questionnaire developed by Marcus et al. (1992). After pilot testing the questionnaire, we added items specific for cancer survivors and arrived at a 14 point scale (Basen-Engquist et al. 2009, 2013). A typical question was: "How confident are you that you can exercise... When you are concerned about your medical condition?" Participants responded to a level of confidence ranging from $1=$ "not at all confident" to $5=$ "extremely confident". Responses to the 14 individual items were summed to obtain an overall score with a possible range of 14-70. Internal consistency (Cronbach's alpha) for our survey was $r=0.83$.

We assessed social support for exercise by adapting Sallis' Social Support and Exercise Survey (Sallis et al. 1987) to a 10-item survey. Participants were asked about the degree of support for exercise that they receive from family/friends. A typical question was: "During the past 3 months, my family (or members of my household) helped plan activities around my exercise". Response categories included: $1=$ "none", 2 = "rarely", 3 = "few times", $4=$ "often" and $5=$ "very often". We analyzed responses related to social support from family separate from social support from friends. Responses to the individual items were summed to obtain an overall score with a possible range of 10-50. Internal consistency (Cronbach's alpha) for our surveys were $r=0.90$ and $r=0.94$ for social support from family and friends, respectively.

Modeling of physical activity was assessed with eight questions that asked the participants to indicate whether they observed people in their social environment or the media engaging in or discussing physical activity/exercise. Participants were asked to respond "yes" or "no" to the following questions: "Today, the following happened: (1) I noticed people like me exercising; (2) A friend or family member offered to exercise with me; (3) I read or heard news stories about people exercising; (4) I was aware that a member of my family exercised today; (5) A friend or family member exercised with me; (6) A friend or family member talked to me about their exercise program; (7) A friend or family member shared their experience about how to stay with an exercise program; and (8) I noticed people walking in my neighborhood." The total number of "yes" responses was added to obtain a total score with a possible range from 0 to 8 . Internal consistency (Cronbach's alpha) for our survey was $r=0.75$.

Upon completion of the questionnaires, height $(\mathrm{m})$, weight $(\mathrm{kg})$, waist circumference $(\mathrm{cm})$ and hip circumference $(\mathrm{cm})$ were measured for each participant. Height 
(m) and weight $(\mathrm{kg})$ data were used to calculate BMI $(\mathrm{kg} /$ $\mathrm{m}^{2}$ ). (At UPRCC, self-reported height and weight were used for BMI calculations instead of being measured). Waistline measurements were taken at the midpoint between the lowest rib and the iliac crest, at the iliac crest, at the narrowest point of the waist area, over the umbilicus area, and at the widest part of the hips. When the tape was accurately placed on the anatomical site against the skin, the participant was asked to inhale and exhale. The measurement was then taken in inches after the participant exhaled. Inches were converted to $\mathrm{cm}$ for the narrowest part of the waist and for the widest part of the hips. A waist-to-hip (W:H) ratio was calculated.

\section{Treatment of data}

All data were entered and analyzed using SPSS V 20.0 (Statistical Package for the Social Sciences), (SPSS, Inc., Chicago, Ill, USA). Descriptive statistics were calculated for participant demographics, activity levels, SCT variables, and anthropometric measures. To compare mean differences among the three groups, we conducted a oneway analysis of variance (ANOVA) with a post-hoc Tukey analysis. We then ran a two-step regression model for current level of activity (GLTEQ), first with demographic variables (education, age, and ethnic group) and then with the SCT variables (exercise barriers self-efficacy, exercise self-efficacy, social support from family, social support from friends, and modeling) added as predictors; changes in $R^{2}$ were calculated. We then ran models that added the interaction terms between each SCT variable and ethnic group. Statistical significance for ANOVA differences and regression analyses was set at $p>0.05$.

\section{Results}

Participant demographic and health characteristics are shown in Table 1. There were no differences among groups in age, BMI and co-morbidity index. Averaged together our participants averaged 56.8 years of age, an overweight BMI of $29.0 \mathrm{~kg} / \mathrm{m}^{2}$ and moderate level of 2.1 co-morbidities when measured with our index. The NHW group was more educated than either the PR or MA subgroups, with more NHW participants reporting being employed. Waist-to-hip ratio were similar for PR and MA ( $\mathrm{M}=0.83, \mathrm{M}=0.82$, respectively) and lower for NHW $(\mathrm{M}=0.79)$.

Results of analyses of frequencies of intensity levels of PA and calculated GLTEQ scores are shown in Table 2. Differences are apparent in the GLTEQ score $(p<0.001)$ with the PR group having the lowest levels $(M=12.9$, $\mathrm{SD}=17.6)$, MA higher than PR level $(\mathrm{M}=31.4$, $\mathrm{SD}=18.7)$ and NHW demonstrating the highest levels $(\mathrm{M}=40.9, \mathrm{SD}=25.9)$ of the three groups. The

Table 1 Comparison of demographic and health variables for Puerto Rican, Mexican-American and non-Hispanic white breast cancer survivors

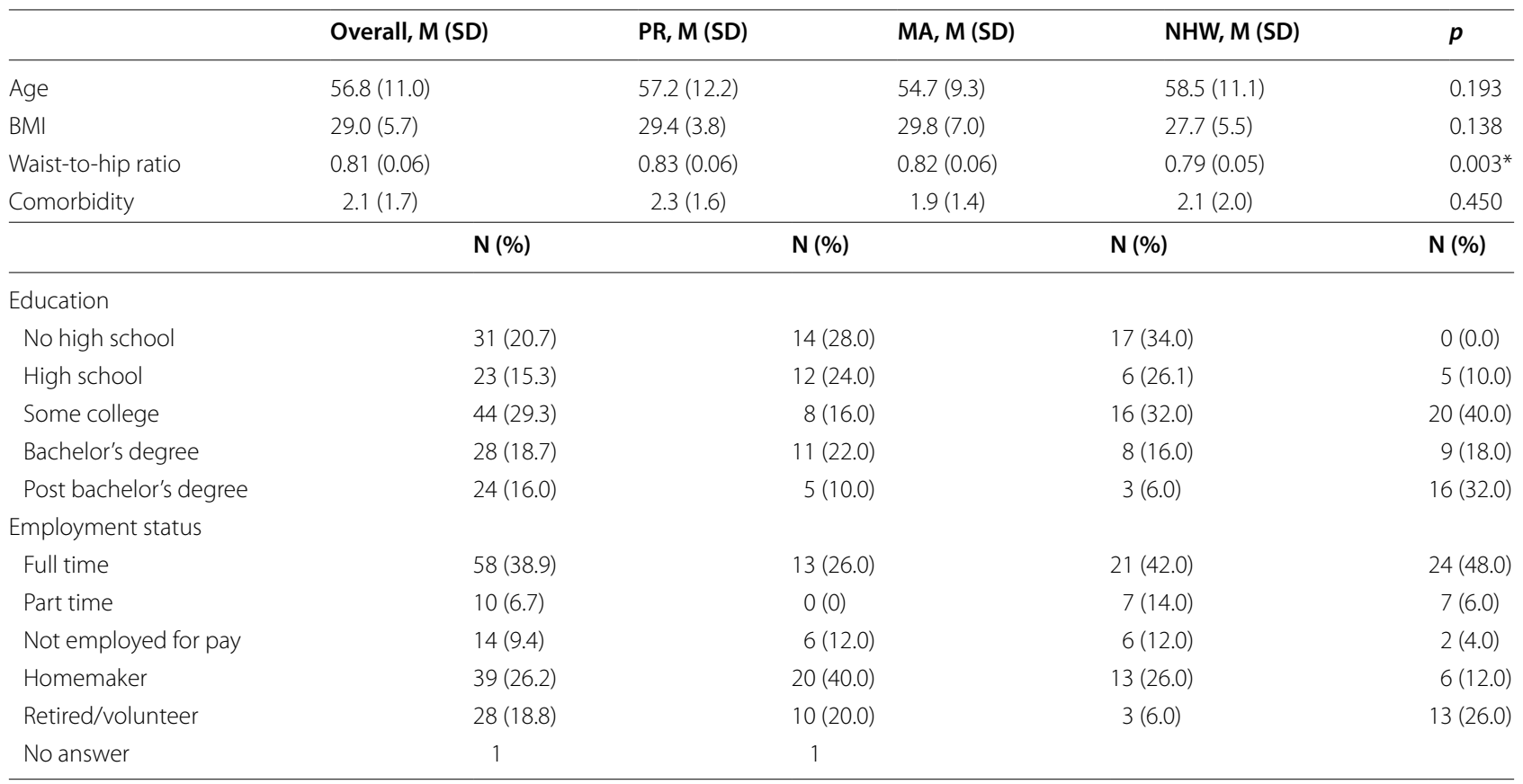

PR Hispanic Puerto Rican participants, MA Hispanic Mexican-American participants, NHW non-Hispanic white participants, SD standard deviation.

* PR > NHW, $(p=0.006)$; MA > NHW, $(p=0.026)$. 
Table 2 Descriptive statistics for Godin Leisure Time Activity Scale by ethnic group

\begin{tabular}{|c|c|c|c|}
\hline & Puerto Rican & $\begin{array}{l}\text { Mexican- } \\
\text { American }\end{array}$ & $\begin{array}{l}\text { Non-Hispanic } \\
\text { white }\end{array}$ \\
\hline \multicolumn{4}{|c|}{ Light activity frequency } \\
\hline$\%$ with no activity & 62 & 18 & 18 \\
\hline Quartiles & $0,0,3$ & $1,4,7$ & $1.75,3,7$ \\
\hline Mean (SD) & $1.76(2.49)$ & $3.82(2.70)$ & $3.60(2.63)$ \\
\hline \multicolumn{4}{|c|}{ Moderate activity frequency } \\
\hline$\%$ with no activity & 76 & 28 & 26 \\
\hline Quartiles & $0,0,0.25$ & $0,2,3$ & $0,2.50,5$ \\
\hline Mean (SD) & $1.06(2.20)$ & $2.12(1.85)$ & $2.78(2.30)$ \\
\hline \multicolumn{4}{|c|}{ Strenuous activity frequency } \\
\hline$\%$ with no activity & 94 & 60 & 44 \\
\hline Quartiles & $0,0,0$ & $0,0,2$ & $0,1,3$ \\
\hline Mean (SD) & $0.26(1.06)$ & $1.04(1.67)$ & $1.8(1.95)$ \\
\hline \multicolumn{4}{|c|}{ Moderate + strenuous activity frequency } \\
\hline$\%$ with no activity & 76 & 22 & 18 \\
\hline Quartiles & $0,0,0.25$ & $1,3,5$ & $2,4,7$ \\
\hline Mean (SD) & $1.32(2.64)$ & $3.16(2.63)$ & $4.58(3.32)$ \\
\hline \multicolumn{4}{|c|}{ Godin Leisure-time Activity Score } \\
\hline$\%$ with score of 0 & 44 & 4 & 2 \\
\hline Quartiles & $0,9,21$ & $18,33,45$ & $21,39,63$ \\
\hline Mean (SD) & $13.98(19.16)$ & $33.54(19.81)$ & $43.68(27.59)$ \\
\hline
\end{tabular}

differences remained significant after controlling for age, education, and employment status $(\mathrm{p}<0.001)$. Seventysix percent of PR survivors reported doing no moderate or strenuous activity compared to $22 \%$ and $18 \%$ of MA and NHW participants, respectively.

Means and standard deviations for the SCT variables are presented in Table 3. A difference in exercise self-efficacy approached statistical significance
( $p=0.05)$, and NHW had marginally higher self-efficacy $(\mathrm{M}=26.0)$ compared to $\mathrm{MA}(\mathrm{M}=21.7 ; p=0.076)$ and PR $(M=22.4, p=0.164)$. Social support from family members differed among the groups $(p=0.052)$ and approached statistical significance, MA survivors reported more social support from family $(\mathrm{M}=25.4)$ than did NHW ( $\mathrm{M}=22.4, p=0.336)$ and PR survivors reported the least social support from family $(M=20.4$; $\mathrm{p}=0.055)$. Significant differences were apparent among the groups in their reporting of exposure to modeling of PA $(\mathrm{p}=0.034)$ with PR survivors reporting significantly higher levels of modeling $(M=3.3)$ than did NHW $(\mathrm{M}=2.22, \mathrm{p}=0.036)$ and higher levels of modeling than $\operatorname{did} \mathrm{MA}(\mathrm{M}=2.64, p=0.272)$. When we controlled for age, education, and employment status, the group differences for exercise self-efficacy and social support from family were somewhat attenuated $(\mathrm{p}=0.286$ and 0.118 , respectively), whereas the effect of modeling remained statistically significant $(\mathrm{p}=0.044)$.

In the regression model, predicting GLTEQ score with the predictor variables of ethnicity, age, and education (step 1), and exercise barriers self-efficacy, exercise self-efficacy, social support from family, social support from friends, and modeling entered as predictors (step 2 ), demographic variables predicted $26 \%$ of the variance in GLTEQ score $(\mathrm{F}=9.9$ [5,142], $\mathrm{p}<0.001)$ and step 2 (SCT) variables predicted an additional $17 \%$ of the variance $(\mathrm{F}=8.2[5,137], \mathrm{p}<0.001)$ for a total explained variance of $43 \%$. Significant predictors in this model were MA ethnicity $(\beta=-0.18, t=-2.1, p=0.040)$, PR ethnicity $(\beta=-0.54, \mathrm{t}=-6.5, \mathrm{p}<0.001)$, exercise barriers self-efficacy ( $\beta=0.21, \mathrm{t}=2.6, \mathrm{p}=0.012)$, and modeling $(\beta=0.22, \mathrm{t}=2.8, \mathrm{p}=0.005)$.

When the interaction terms between ethnic group and each SCT variable were added to the model one SCT

Table 3 Comparisons of SCT variables assessed in Puerto Rican, Mexican-American and Non-Hispanic white breast cancer survivors

\begin{tabular}{|c|c|c|c|c|c|c|}
\hline & Overall, M (SD) & PR, M (SD) & $M A, M(S D)$ & NHW, M (SD) & ANOVA, $p$ & Post-hoc, ' $t$ ' \\
\hline Barriers self-efficacy & $38.6(12.5)$ & $36.9(13.9)$ & $38.5(9.1)$ & $40.5(13.8)$ & 0.370 & \\
\hline Exercise self-efficacy & $23.4(9.4)$ & $22.4(11.2)$ & $21.7(7.6)$ & $26.0(8.7)$ & 0.052 & $\begin{array}{l}N H W>M A(p=0.076) \\
N H W>\operatorname{PR}(p=0.164)\end{array}$ \\
\hline Social support-friends & $20.8(12.0)$ & $18.7(10.8)$ & $23.5(13.9)$ & $20.2(10.6)$ & 0.120 & \\
\hline Social support-family & $22.7(10.4)$ & $20.4(11.5)$ & $25.4(9.7)$ & $22.3(9.4)$ & 0.052 & $\begin{array}{l}\mathrm{MA}>\mathrm{PR}(p=0.055) \\
\mathrm{MA}>\mathrm{NHW}(p=0.336)\end{array}$ \\
\hline Modeling & $2.73(2.14)$ & $3.32(2.37)$ & $2.64(2.07)$ & $2.22(1.87)$ & 0.034 & $\begin{array}{l}\mathrm{PR}>\operatorname{NHW}(p=0.036) \\
\mathrm{PR}>\mathrm{MA}(p=0.272)\end{array}$ \\
\hline GLTEQ & $28.4(23.9)$ & $12.9(17.6)$ & $31.4(18.7)$ & $40.9(25.9)$ & $<0.001$ & $\begin{array}{l}N H W>P R(p<0.001) \\
M A>P R(p<0.001) \\
N H W>M A(p=0.083)\end{array}$ \\
\hline
\end{tabular}

Italic values are statistically significant $(p<0.05)$.

$P R$ Hispanic Puerto Rican participants, MA Hispanic Mexican-American participants, NHW non-Hispanic white participants, $M$ mean, $S D$ standard deviation, GLTEQ Godin Leisure Time Exercise Questionnaire Score. 
variable at a time, the only interactions that were significant were those between ethnic group and exercise barriers self-efficacy, and this explained an additional $4 \%$ of the variance in GLTEQ score (F-change $=4.6[2,135]$, $\mathrm{p}=0.012$ ). In this model, the interactions between exercise barriers self-efficacy and MA (beta $=-0.77$, $\mathrm{t}=-2.7, \mathrm{p}=0.007$ ) and PR (beta $=-0.51, \mathrm{t}=-2.3$, $\mathrm{p}=0.024)$ ethnicity were significant, indicating that exercise barriers self-efficacy was less predictive of GLTEQ score for MA and PR than it was for NHW.

\section{Discussion}

Of the more than 12 million cancer survivors in the United States, approximately 15\% are Hispanic (American Cancer Society 2009). The Latino population is the most rapidly growing ethnic group in the United States (United States Census Bureau 2000; Census Bureau 2011). As current trends continue, the number of Latina women with breast cancer in the US population will escalate just as dramatically (American Cancer Society 2009).

Consistent with published studies in Hispanic populations not affected by cancer (Cantero et al. 1999; Abraido-Lanza et al. 2005), our study indicates that Hispanic breast cancer survivors are less physically active than are non-Hispanic whites. Our study also was consistent with published studies that have shown higher BMI and waist-to-hip ratios for Latinas versus non-Hispanic whites (Hubert et al. 2005; Ogden et al. 2006; Slattery et al. 2006).

The percentage of breast cancer survivors reporting no moderate- or vigorous-intensity physical activity ranges from 18 to $76 \%$, depending on ethnic group. The inactivity rates of PR survivors were markedly higher than those of the MA and NHW survivors. This is in contrast to data from the Behavioral Risk Factor Surveillance System indicating that rates of no exercise in the past 30 days are similar in Texan and Puerto Rican Hispanics (approximately $38 \%$ ). In this survey, the Puerto Rican survivors' median GLTEQ index was 9.0 (25th percentile $=0$, 75th percentile $=21)($ Center for Disease Control and Prevention 2012), indicating a very low level of current physical activity. The fact that $76 \%$ of our PR survivors indicate no moderate- to vigorous-intensity activity indicates that only a small proportion $(<24 \%)$ of the respondents are meeting American Cancer Society physical activity guidelines for cancer survivors (Rock et al. 2012). This level of physical activity is lower than that reported by breast cancer survivors meeting physical activity recommendations in a survey of cancer survivors from a crosssectional survey of cancer survivors in 16 state cancer registries (American Cancer Society's Study of Cancer Survivors-II; ACS SCS-II). In this study (which had fewer than $10 \%$ Hispanic participants), $37 \%$ of the breast cancer survivors were meeting physical activity recommendations (Blanchard et al. 2008).

These very low activity levels could be attributable to several factors. In focus groups conducted with Puerto Rican and Mexican-American breast cancer survivors, we found that both groups lacked knowledge of the safety of physical activity after breast cancer. Although participants reported being physically active before their diagnosis, many reported that they did not know what they could or could not do after treatment. Focus group members expressed a desire for more information but few reported receiving any guidance or direction from health care providers with regard to physical activity. This situation was seemingly validated by modeling, as many of the breast cancer survivors reported being unaware of other cancer survivors engaging in exercise. In addition, many of our focus group participants reported that their family members did not encourage them to engage in exercise (Trevino et al. 2012). We also found that participants lacked knowledge about the benefits of physical activity; the Puerto Rican focus groups, in particular, mentioned several times that they did not think that physical activity could help prevent cancer. Because they did not see a link between physical activity and cancer, being active may have had less salience for the Puerto Rican survivors (Trevino et al. 2012).

Additionally, both groups reported a variety of other barriers to exercise. When asked about safety in their neighborhoods, Puerto Ricans stating they had little or no safety concerns, whereas the Mexican-American groups did report safety concerns as a barrier for physical activity. Both groups also mentioned social support as a barrier for physical activity, with Puerto Ricans stating that they would feel more motivated to be active if they had someone to exercise with that someone not necessarily having to be a family member (Trevino et al. 2012).

Furthermore, the PR survivors were recruited for this study in a public hospital in metropolitan San Juan Puerto Rico, and the MA and NHW participants were recruited through MD Anderson Cancer Center and from support groups in the Houston area. It is possible that the PR survivors were of lower socioeconomic status (SES), and this might help explain the lower rates of physical activity, although the differences in activity levels were not attenuated by controlling for education.

Our study also indicated that the activity-related SCT variables differed between Hispanic breast cancer survivor groups and non-Hispanic whites as has been reported in other studies (Whitehorse et al. 1999), but we also found a difference within Hispanic groups. MA survivors reported being less confident that they would be able to complete walking sessions of various lengths than did NHW survivors, and PR survivors 
reported seeing more people in their environment doing physical activity than did MA survivors. This difference may relate back to neighborhood safety issues in the MA group. Another difference between the two Hispanic groups was that social support for physical activity from family was higher in MA than in the PR women. This could be attributable to different cultural norms about exercise for cancer survivors in the two locations, although more research is needed to understand these cultural norms in promoting physical activity.

In associations between the SCT variables and leisure time physical activity, the set of five SCT variables predicted an additional $17 \%$ of the variance in the physical activity score. This percentage was above and beyond the effect of the demographic variables alone. Exercise barriers self-efficacy and modeling were significantly associated with activity levels. However, it should be noted that the association of barrier self-efficacy with activity was significantly smaller in the two Hispanic groups. Studies in other cancer survivor populations also have found associations between exercise barriers self-efficacy and physical activity, but these studies did not include large numbers of Hispanic survivors (Rogers et al. 2005; Bennett et al. 2007; Jones et al. 2005; Mosher et al. 2008; Pinto et al. 2009; Vallance et al. 2008).

Additional research is needed to determine why Hispanic survivors lack the confidence that one can overcome barriers to being consistently active and why they may believe exercise is less important. It is also important to understand how this information can be used further in culturally adapting interventions. The finding that modeling is a correlate of leisure time physical activity in these breast cancer survivors is somewhat novel, as this variable has not been routinely measured in many studies. A notable exception is a study by Rogers and colleagues, which showed that having an exercise role model was associated with steps taken and caloric expenditure in a sample of 21 breast cancer survivors receiving treatment (Rogers et al. 2005).

This study has certain limitations that should be noted. Because the sample size is modest, some of the lack of significant differences among the three groups in the SCT variables may be due to a lack of statistical power. The recruitment setting in Puerto Rico differed from that of the Mexican-American and non-Hispanic white populations and this may have accounted for some of the differences observed. We controlled for education level as a measure of SES status, but future studies should use additional measures of SES. In addition, because the sample is cross-sectional, the associations between the SCT variables and activity may not be causal. Further research is necessary to determine whether providing models for activity and improving barriers self-efficacy are effective tools for increasing physical activity in Hispanic breast cancer survivors. However, this study does represent one of the first attempts to measure and analyze social cognitive theory variables and leisure time physical activity in a sample of breast cancer survivors from two different Hispanic cultures. This study also had a comparison group of non-Hispanic white survivors that provided important information about future research directions.

\section{Conclusions}

Further research aimed at understanding culturally specific variables of exercise behaviors for ethnic breast cancer survivors is warranted. Culturally adapted intervention strategies are needed for increasing physical activity in specific ethno-cultural populations.

\section{Abbreviations}

PA: physical activity; PR: Puerto Rican; MA: Mexican-American; NHW: non-Hispanic white; SCT: social cognitive theory; GLTEQ: Godin Leisure Time Exercise Questionnaire; BMI: body mass index; W:H: waist-to-hip; M: mean; SD: standard deviation; BRTC: Behavioral Research and Treatment Center; UTMDACC: University of Texas M. D. Anderson Cancer Center; UPRCC: University of Puerto Rico Cancer Center; MET: metabolic equivalent; SPSS: statistical package for the social sciences; ANOVA: analysis of variance; SES: socioeconomic status; PROSPR: patient-reported outcomes, survey, and population research.

\section{Authors' contributions}

DCH designed and managed the study, trained the project coordinators on data collection, ran the data analyses, drafted the manuscript and participated in interpretation of the results. MTG co-designed the overall study, managed the data collection in Puerto Rico, interpreted the Puerto Rico results and co-wrote the discussion section. LV managed that Texas site data collection, wrote the Texas data collection methodology sections and helped interpret the results. VG managed the Puerto Rico site data collection, validated all translations, wrote the Puerto Rico data collection methodology and helped interpret the results. RTW validated translations, co-wrote the Texas data collection methodology, helped with the data analyses and interpret the results. GV helped with the data analyses, co-wrote the background section and helped interpret the results. KBE co-designed and co-managed the overall study, oversaw all aspects of the manuscript, and led the interpretation of the results. All authors read and approved the final manuscript.

\section{Author details \\ ${ }^{1}$ Institute for Health Promotion Research, The University of Texas Health Sci- ence Center at San Antonio, 7411 John Smith Drive, Suite 1000, San Antonio, TX 78229, USA. ${ }^{2}$ The University of Puerto Rico Cancer Center, San Juan, Puerto Rico. ${ }^{3}$ Department of Behavioral Science, The University of Texas M.D. Ander- son Cancer Center, Houston, USA.}

\section{Acknowledgements}

The authors would like to acknowledge all the participants for their commitment to the study. The project described was supported by award numbers K22CA154626, U54 CA153511, U54CA96297 and U54CA96300 from the National Cancer Institute of the National Institutes of Health. The content is solely the responsibility of the authors and does not necessarily represent the official views of the National Cancer Institute or the National Institutes of Health. The authors gratefully acknowledge the support of the Patient-Reported Outcomes, Survey, and Population Research (PROSPR) Shared Resource, National Cancer Institute funded CA16672 and the Cancer Therapy and Research Center at The University of Texas Health Science Center at San Antonio, a National Cancer Institute-designated Cancer Center (P30CA054174). 


\section{Compliance with ethical guidelines}

\section{Competing interests}

The authors confirm that this study was performed with the highest ethical standards and complying with all United States laws. The authors declare that they have no competing interests. The authors have full control of all primary data.

\section{Ethical approval}

All procedures performed in studies involving human participants were in accordance with the ethical standards of the institutional and/or national research committee and with the 1964 Helsinki declaration and its later amendments or comparable ethical standards.

\section{Informed consent}

Informed consent was obtained from all individual participants included in the study.

Received: 25 July 2015 Accepted: 29 July 2015

Published online: 13 August 2015

\section{References}

Abraido-Lanza AF, Chao MT, Florez KR (2005) Do healthy behaviors decline with greater acculturation? Implications for the Latino mortality paradox. Soc Sci Med 61 (6):1243-1255

American Cancer Society (2009) Cancer facts and figures for Hispanics/Latinos. 2009-2011. American Cancer Society, Atlanta

American Cancer Society (2012) Cancer facts \& figures 2012. American Cancer Society, Atlanta

Ballard-Barbash R, Friedenreich CM, Courneya KS, Siddiqi SM, McTiernan A, Alfano CM (2012) Physical activity, biomarkers, and disease outcomes in cancer survivors: a systematic review. J Natl Cancer Inst 104(11):815-840. doi:10.1093/jnci/djs207

Basen-Engquist K, Baum G, Hughes DC, Scruggs S, Carmack Taylor C (2009) Validity and reliability of a cancer survivors' exercise barriers self-efficacy scale. Ann Behav Med 37 (Suppl):S185 (\#D-107)

Basen-Engquist K, Carmack CL, Li Y, Brown J, Jhingran A, Hughes DC et al (2013) Social-cognitive theory predictors of exercise behavior in endometrial cancer survivors. Health Psychol. doi:10.1037/a0031712

Bennett JA, Lyons KS, Winters-Stone K, Nail LM, Scherer J (2007) Motivational interviewing to increase physical activity in long-term cancer survivors: a randomized controlled trial. Nurs Res 56(1):18-27 (pii: 00006199-200701000-00003)

Blanchard CM, Courneya KS, Stein K, American Cancer Society's SCS, II (2008) Cancer survivors' adherence to lifestyle behavior recommendations and associations with health-related quality of life: results from the American Cancer Society's SCS-II. J Clin Oncol 26(13):2198-2204. doi:10.1200/ JCO.2007.14.6217

Cantero PJ, Richardson JL, Baezconde-Garbanati L, Marks G (1999) The association between acculturation and health practices among middle-aged and elderly Latinas. Ethn Dis 9(2):166-180

Castro C, Sallis J, Hickman S, Lee RE, Chen AH (1999) A prospective study of psychosocial correlates of physical activity for ethnic minority women. Psychol Health 14:277-293

Center for Disease Control and Prevention (2012) Behavioral risk factor surveillance system. http://www.cdc.gov/brfss/. Accessed 31 Aug 2012

Courneya KS (2003) Exercise in cancer survivors: an overview of research. Med Sci Sports Exerc 35(11):1846-1852

Courneya KS, Mackey JR, Bell GJ, Jones LW, Field CJ, Fairey AS (2003) Randomized controlled trial of exercise training in postmenopausal breast cancer survivors: cardiopulmonary and quality of life outcomes. J Clin Oncol 21(9):1660-1668

Crespo CJ, Smit E, Andersen RE, Carter-Pokras O, Ainsworth BE (2000) Race/ ethnicity, social class and their relation to physical inactivity during leisure time: results from the Third National Health and Nutrition Examination Survey, 1988-1994. Am J Prev Med 18(1):46-53 (pii: S0749-3797(99)00105-1)
Duncan TE, McAuley E (1993) Social support and efficacy cognitions in exercise adherence: a latent growth curve analysis. J Behav Med 16(2):199-218

Godin G, Shephard RJ (1985) A simple method to assess exercise behavior in the community. Can J Appl Sport Sci 10(3):141-146

Holmes MD, Chen WY, Feskanich D, Kroenke CH, Colditz GA (2005) Physical activity and survival after breast cancer diagnosis. JAMA 293(20):24792486. doi:10.1001/jama.293.20.2479

Hubert HB, Snider J, Winkleby MA (2005) Health status, health behaviors, and acculturation factors associated with overweight and obesity in Latinos from a community and agricultural labor camp survey. Prev Med 40(6):642-651

Irwin ML, McTiernan A, Manson JE, Thomson CA, Sternfeld B, Stefanick ML et al (2011) Physical activity and survival in postmenopausal women with breast cancer: results from the women's health initiative. Cancer Prev Res (Phila) 4(4):522-529. doi:10.1158/1940-6207.CAPR-10-0295

Jones LW, Courneya KS, Fairey AS, Mackey JR (2005) Does the theory of planned behavior mediate the effects of an oncologist's recommendation to exercise in newly diagnosed breast cancer survivors? Results from a randomized controlled trial. Health Psychol 24(2):189-197. doi:10.1037/0278-6133.24.2.189

Marcus BH, Selby VC, Niaura RS, Rossi JS (1992) Self-efficacy and the stages of exercise behavior change. Res Q Exerc Sport 63(1):60-66

Mason C, Alfano CM, Smith AW, Wang CY, Neuhouser ML, Duggan C et al (2013) Long-term physical activity trends in breast cancer survivors. Cancer Epidemiol Biomarkers Prev. doi:10.1158/1055-9965.EPI-13-0141

McAuley E (1993) Self-efficacy and the maintenance of exercise participation in older adults. J Behav Med 16(1):103-113

McAuley E, Courneya KS, Rudolph DL, Lox CL (1994) Enhancing exercise adherence in middle-aged males and females. Prev Med 23(4):498-506

McAuley E, Jerome GJ, Marquez DX, Elavsky S, Blissmer B (2003) Exercise selfefficacy in older adults: social, affective, and behavioral influences. Ann Behav Med 25(1):1-7

Mosher CE, Fuemmeler BF, Sloane R, Kraus WE, Lobach DF, Snyder DC et al (2008) Change in self-efficacy partially mediates the effects of the FRESH START intervention on cancer survivors' dietary outcomes. Psycho-oncology 17(10):1014-1023. doi:10.1002/pon.1327

Ogden CL, Carroll MD, Curtin LR, McDowell MA, Tabak CJ, Flegal KM (2006) Prevalence of overweight and obesity in the United States, 1999-2004. JAMA 295(13):1549-1555

Patterson RE, Cadmus LA, Emond JA, Pierce JP (2010) Physical activity, diet, adiposity and female breast cancer prognosis: a review of the epidemiologic literature. Maturitas 66(1):5-15. doi:10.1016/.maturitas.2010.01.004

Pinto BM, Frierson GM, Trunzo JJ, Marcus BH (2005) Home-based physical activity intervention for breast cancer patients. J Clin Oncol 23(15):3577-3587

Pinto BM, Rabin C, Dunsiger S (2009) Home-based exercise among cancer survivors: adherence and its predictors. Psycho-oncology 18(4):369-376 doi:10.1002/pon. 1465

Puerto Rico Central Cancer Registry (2007) Puerto Rico Cancer Incidence File (May 2007)

Rock CL, Doyle C, Demark-Wahnefried W, Meyerhardt J, Courneya KS, Schwartz AL et al (2012) Nutrition and physical activity guidelines for cancer survivors. CA Cancer J Clin 62(4):243-274. doi:10.3322/caac.21142

Rogers LQ, Shah P, Dunnington G, Greive A, Shanmugham A, Dawson B et al (2005) Social cognitive theory and physical activity during breast cancer treatment. Oncol Nurs Forum 32(4):807-815. doi:10.1188/04.ONF.807-815

Sallis JF, Grossman RM, Pinski RB, Patterson TL, Nader PR (1987) The development of scales to measure social support for diet and exercise behaviors. Prev Med 16(6):825-836

Schmitz KH, Courneya KS, Matthews C, Demark-Wahnefried W, Galvao DA, Pinto BM et al (2010) American College of Sports Medicine roundtable on exercise guidelines for cancer survivors. Med Sci Sports Exerc 42(7):1409-1426

Segal R, Evans W, Johnson D, Smith J, Colletta S, Gayton J et al (2001) Structured exercise improves physical functioning in women with stages I and II breast cancer: results of a randomized controlled trial. J Clin Oncol 19(3):657-665

Slattery ML, Sweeney C, Edwards S, Herrick J, Murtaugh M, Baumgartner Ket al (2006) Physical activity patterns and obesity in Hispanic and non-Hispanic white women. Med Sci Sports Exerc 38(1):33-41 (pii: 00005768-200601000-00007) 
Trevino RA, Vallejo L, Hughes DC, Gonzalez V, Tirado-Gomez M, Basen-Engquist K (2012) Mexican-American and Puerto Rican breast cancer survivors' perspectives on exercise: similarities and differences. J Immigr Minor Health. doi:10.1007/s10903-012-9648-9

United States Census Bureau (2000) Statistical abstract of the United States: 2000, 120th edn. United States Government Printing Office, Washington, DC

U.S. Census Bureau (2011) 2011 Census Briefs The Hispanic Population: 2010. http://www.census.gov/prod/cen2010/briefs/c2010br-04.pdf. Accessed 03 Dec 2012
Vallance JK, Courneya KS, Plotnikoff RC, Mackey JR (2008) Analyzing theoretical mechanisms of physical activity behavior change in breast cancer survivors: results from the activity promotion (ACTION) trial. Ann Behav Med 35(2):150-158. doi:10.1007/s12160-008-9019-x

Whitehorse LE, Manzano R, Baezconde-Garbanati LA, Ginger H (1999) Culturally tailoring a physical activity program for Hispanic women: recruitment successes of La Vida Buena's salsa aerobics. J Health Educ 30(2):S18-S24

\section{Submit your manuscript to a SpringerOpen ${ }^{\odot}$ journal and benefit from:}

- Convenient online submission

- Rigorous peer review

- Immediate publication on acceptance

- Open access: articles freely available online

- High visibility within the field

- Retaining the copyright to your article

Submit your next manuscript at $>$ springeropen.com 